EPJ manuscript No.

(will be inserted by the editor)

\title{
Coherent photon-photon interactions in very peripheral relativistic heavy ion collisions
}

\author{
Gerhard Baur ${ }^{1,2}$ \\ 1 Institut für Kernphysik, Forschungszentrum Jülich, D-52425 Jülich, Germany \\ 2 Jülich Centre for Hadron Physics, Forschungszentrum Jülich, D-52425 Jülich, Germany \\ Received: date / Revised version: date
}

\begin{abstract}
Heavy ions at high velocities provide very strong electromagnetic fields for a very short time. The main characteristics of ultraperipheral relativistic heavy ion collisions are reviewed, characteristic parameters are identified. The main interest in ultraperipheral heavy ion collisions at relativistic ion colliders like the LHC is the interactions of very high energy (equivalent) photons with the countermoving (equivalent) photons and hadrons (protons/ions). The physics of these interactions is quite different from and complementary to the physics of the strong fields achieved with current and future lasers.
\end{abstract}

PACS. 25.75.Ag Global features in relativistic heavy ion collisions

\section{Introduction}

Strong fields are a probe for fundamental physics. Modern lasers provide very strong fields. In this context it is interesting to ask what is the characteristics of strong fields occurring in relativistic heavy ion collisions. In this contribution the physics of ultraperipheral relativistic heavy ion collisions is described. The fields are extremely strong, typically much stronger than the Schwinger critical field. On the other hand these fields act only for an extremely short time. There are comprehensive recent reviews on ultraperipheral heavy ion collisions 1, 2. Nevertheless it is of interest to give an overview of key physics aspects in a qualitative and 'context-setting' way. The extremely short duration of these pulses leads to a clear difference to strong field physics in lasers.

Electric fields of heavy ions are rather strong, a good measure is the quantity $Z \alpha$, where $\alpha \equiv \frac{e^{2}}{\hbar c} \sim 1 / 137$ is the fine structure constant. For $\mathrm{Pb}$ we have $Z=82$ and $Z \alpha \sim$ 0.6. In slow $(v<<c)$ heavy ion collisions a united atom is formed for an appreciable time where $Z_{1}+Z_{2}>Z_{\text {overcritical }}$ is possible. For $Z>Z_{\text {overcritical }} \sim 173$ the $s_{1 / 2}$-state dives into the negative energy continuum. In this contribution the opposite limit $v \sim c$ and $\gamma=1 / \sqrt{1-(v / c)^{2}}>>1$ is assumed.

In Sect. 2 the characteristics of the fast and strong electromagnetic pulse are described as well as the transition from fast to slow heavy ion collisions. This transition is controlled by a parameter related to the Keldysh parameter in atomic physics. Then it is shown how to describe theoretically one- and multi-photon processes. A very convenient tool to describe one-photon exchange processes is

Send offprint requests to: Gerhard Baur the equivalent photon approximation. Multi-photon processes are treated in the Glauber approximation. Typical examples of $\gamma$-nucleus, $\gamma-$ proton, and $\gamma-\gamma$ collisions at hadron colliders are described. In Sect. 4 higher order processes are considered. Multiphoton processes are especially prominent in electron-positron pair production. This is due to the low mass of the electron. We briefly discuss bound-free pair production and $e^{+} e^{-}$multiple pair production. A conclusion is given in Sect. 4.

\section{Photoproduction in ultraperipheral hadron-hadron collisions}

\subsection{Characteristics of the electromagnetic pulse}

In Fig.1 an ultraperipheral collision with impact parameter $\mathrm{b}$ is shown schematically. The projectile with electric charge $Z e$ has a velocity $v \sim c$. A short and strong electromagnetic pulse is produced with the following characteristics. Due to the Lorentz contraction the field is compressed, the maximum field is in the direction perpendicular to the beam velocity and is of the order of $E_{\max } \sim \frac{Z e}{b^{2}} \gamma$, the magnetic field is given by $\boldsymbol{B}_{\perp}=\boldsymbol{v} \times \boldsymbol{E}$. The collision time is of the order of $\Delta t \sim \frac{b}{\gamma c}$ During this time, electrons can travel only a distance of the order of $\Delta l \sim c \Delta t=b / \gamma$. For impact parameters corresponding to the electron Compton wavelength $b \sim \hbar /(m c)$ this distance is $\Delta l \sim \hbar /(m c \gamma)<<386 \mathrm{fm}$. For many purposes, this pulse can be approximated by a $\delta$-function. In this case the perturbation series can be summed by exponentiation. One can neglect time ordering and the S-matrix is given by $S=\exp \left(i \int H_{\text {int }} d t / \hbar\right)$. 


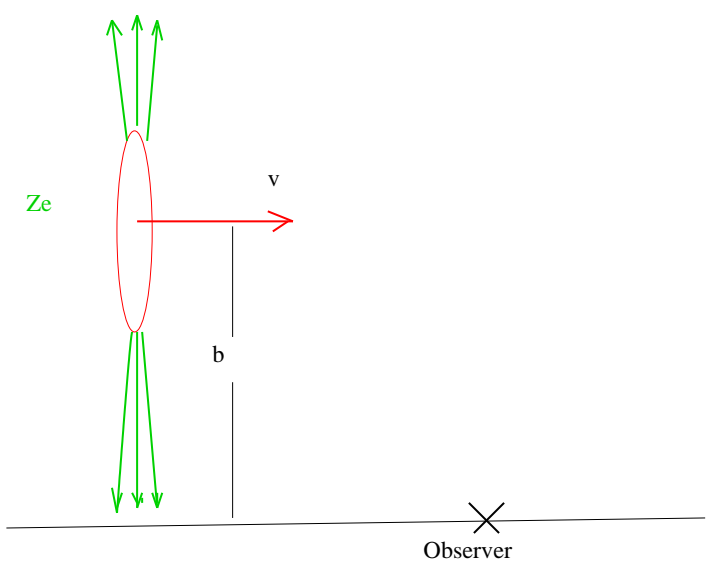

Fig. 1. A schematic picture of an ultraperipheral collision. The impact parameter $\mathrm{b}$ is larger than the sum of the nuclear radii.

As a typical impact parameter we choose the Compton wavelength of the electron. We obtain

$$
E_{\text {max }}=E_{\text {critical }} Z \alpha \gamma
$$

where $E_{\text {critical }}=\frac{m^{2} c^{3}}{\hbar e}=1.3 \times 10^{16} \frac{\mathrm{V}}{\mathrm{cm}}$ is the Schwinger critical field strength. Since $Z \alpha \gamma>>1$ this field strength is much higher than the Schwinger critical field. The corresponding peak power is enormous, it is of the order of $10^{29}(Z \alpha \gamma)^{2} \frac{\mathrm{W}}{\mathrm{cm}^{2}}$, see also [4]. However, the correponding collision time is extremely small, $\tau \sim \frac{\hbar}{m c^{2} \gamma} \sim 10^{-21} \mathrm{~s} / \gamma \equiv$ $1 \mathrm{zs} / \gamma$ (zs=zeptosecond). For RHIC (Au-Au, $\gamma=100)$ this is a small fraction of a zeptosecond, for LHC (p-p, $\gamma=$ 7000 and $\mathrm{Pb}-\mathrm{Pb}, \gamma=3000)$ this is a fraction of $10^{-24} \mathrm{~s} \equiv$ 1ys (yoctosecond). The classical momentum transfer on a test charge e is given by $\Delta p \sim e E_{\max } \tau_{\text {collision }} \sim \frac{Z e^{2}}{b c}$, independent of $\gamma$. On the other hand, the maximum energy of the equivalent photon spectrum rises linearly with $\gamma$ : $E_{\gamma, \max } \sim \frac{\hbar}{\tau_{\text {collision }}} \sim \frac{\hbar c}{b} \gamma$.

In 3] the condition for the electric field to be considered as classical is given by $|\boldsymbol{E}|>>\sqrt{\hbar c} /(c \Delta t)^{2}$. Inserting the above estimates for the field and the collision time for an impact parameter $b=\frac{\hbar}{m c}$ one obtains the condition $Z \sqrt{\alpha}>>\gamma$. For ultrarelativistic collisions $(\gamma>10)$ this cannot be fulfilled, i.e. it is not useful to think of the field as a classical quantity, it is best to think in terms of light quanta (photons), as is usually done anyway.

\subsection{Transition from fast to slow collisions}

Electron-positron pair production in a constant electric field was studied by Schwinger [5]. Pair production becomes appreciable for a field strength larger than the critical field strength $E_{\text {critical }}=\frac{m^{2} c^{3}}{e \hbar}$. The electron mass is denoted by $m$. Electron pair production in the time-varying electric field $\boldsymbol{E}(t)=\boldsymbol{E}_{0} \cos \omega t$ was studied in [6]. A measure of adiabaticity is the parameter $\gamma_{\mathrm{BI}} \equiv \frac{m c \omega}{e E_{0}}=\frac{\omega}{e E_{0}} \frac{\hbar}{\lambda_{e}}$ 6]. This parameter corresponds to the Keldysh parameter $\gamma_{K}=\frac{\omega}{e E_{0}} \frac{\hbar}{a_{\mathrm{Bohr}}}$ in atomic physics. It was shown in [6] that $\gamma_{\mathrm{BI}}<<1$ corresponds to the nonperturbative regime (Schwinger formula). For $\gamma_{\mathrm{BI}}>>1$ the process can be treated perturbatively.

The time- and space-dependence of the electromagnetic field in a heavy ion collision is more complicated than the one envisaged in [6]. Still, one can can find an estimate of this parameter for fast heavy ion collisions. As noted above, the maximum electric field strength is given by $E_{\max }=\frac{Z e}{b^{2}} \gamma$ where $\mathrm{b}$ is the impact parameter and the field strength is appreciable for a time of the order $\Delta t=1 / \omega \sim \frac{b}{\gamma v}$. One finds $\gamma_{\mathrm{HI}}=\frac{m c v b}{Z e^{2}}$. An appropriate minimum impact parameter for processes involving electrons is the Compton wavelength, i.e. $b=\frac{\hbar}{m c}$. in this case one has $\gamma_{\mathrm{HI}}=\frac{v}{c Z \alpha}$. I conclude that relativistic $(v \sim c)$ ultraperipheral reactions can be treated in perturbation theory. The condition is $\frac{v}{c}>Z \alpha$.

\subsection{One- and multi-photon processes, Glauber theory}

One-photon exchange processes are conveniently descibed by the equivalent photon approximation (Weizsäcker- Williams method), see 1 and further references given there. The cross section is obtained as a folding of the equivalent photon spectrum $n(\omega)$ and the elementary photo-cross section $\sigma_{\gamma}(\omega)$ :

$$
\sigma=\int \frac{d \omega}{\omega} n(\omega) \sigma_{\gamma}(\omega)
$$

The equivalent photon spectrum is given approximately by

$$
n(\omega) \sim Z^{2}\left(\frac{c}{v}\right)^{2} \log \frac{\gamma v}{\omega b_{\min }}
$$

The charges of the $\mathrm{Z}$ protons in the nucleus act coherently, this leads to a $Z^{2}$ factor. There are many soft photons and the spectrum extends up to maximum photon energies up to $\omega_{\max }=\frac{\gamma v}{b_{\min }}$. Beyond this energy there is an exponential decrease of the spectrum. For processes involving electrons and positrons a minimum impact parameter is given by $\lambda_{e}=\frac{\hbar}{m_{e} c}$, for other processes an appropriate minimum impact parameter is provided by the nuclear radius $\mathrm{R}$. For RHIC energies $(\gamma=100)$, this maximum energy is about $3 \mathrm{GeV}$, for $\mathrm{LHC}(\mathrm{Pb}-\mathrm{Pb})$ energies $(\gamma=3400)$ it is $100 \mathrm{GeV}$ (in the c.m. (lab)-system).

In slow collisions $(v<<c)$ the electric field acts for a long time and higher order processes (many photon exchanges) are very well known, e.g. the excitation of rotational bands in low energy heavy ion Coulomb exciation. The strength of the interaction decreases with increasing velocity like $1 / v$. Nevertheless many photons can be exchanged even in a relativistic heavy ion collision.

A theoretical tool to describe such many photon exchange processes is the Glauber approximation. The scat- 


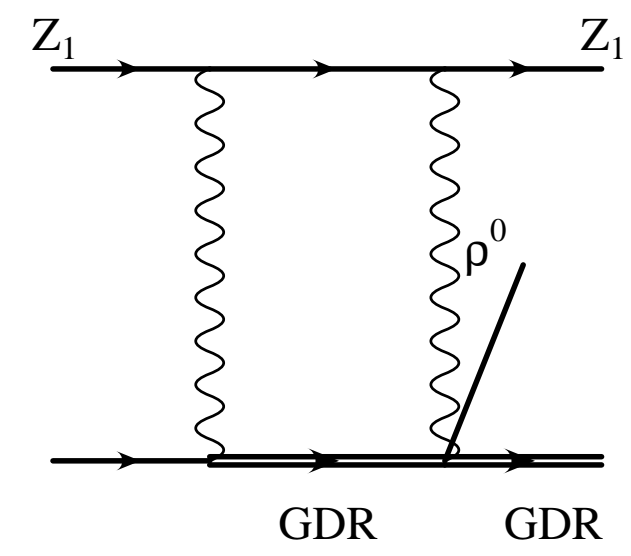

Fig. 2. A second order graph. The giant dipole resonance (GDR) is excited and a $\rho$-meson is produced in a single collision

tering amplitude is given by

$$
f_{f i, \text { Glauber }}=\frac{i \pi}{k} \int d^{2} b \exp (i \boldsymbol{K} \cdot \boldsymbol{b})<f|\exp (i \chi(\boldsymbol{b}))| i>
$$

where $\boldsymbol{K}$ is the momentum transfer. The eikonal $\chi(b)$ takes care of all the elastic and inelastic processes. We have

$$
\chi(b)=\chi(b)_{\mathrm{nuc}}+\chi(b)_{C}+\chi(b)_{e^{+} e^{-}}+\chi(b)_{\mathrm{GDR}}+\chi(b)_{V}+\ldots
$$

The term $\chi_{\text {nuc }}$ describes the effect coming from the nuclear interaction between the two ions. In a sharp cut-off approximation it is given by $\exp \left(i \chi(b)_{n u c}\right)=\Theta(b-2 R)$. The term $\chi_{C}=2 \eta \log (k b)$ describes elastic Coulomb scattering. The Coulomb parameter can be also be much larger than unity for relativistic collisions, i.e. at $\mathrm{LHC}(\mathrm{Pb}-\mathrm{Pb})$ one has $\eta=49$. It means that many 'elastic photons' are exchanged. The term $\chi(b)_{e^{+} e^{-}}$describes electron- positron pair production. The excitation of the giant dipole resonance (GDR), the production of vector mesons $\mathrm{V}(V=$ $\rho, \omega, \phi, J / \Psi, \Upsilon, \ldots)$ is given by the corresponding Glauber phases. In this way, many photon exchange processes, like GDR excitation and vector meson production, etc. can be conveniently described theoretically. This is an efficient way to avoid the evaluation of Feynman diagrams in the limit of high energies, see Figs. 2 and 3. Further details can be found in [7, 8].

\section{$2.4 \gamma-\mathbf{A}, \gamma-\mathbf{p}$, and $\gamma-\gamma$ interactions in ultraperipheral hadron-hadron collisions}

Even in the very fast relativistic heavy ion collisions multiphoton processes occur. A good example is the excitation of the double phonon giant dipole resonance at GSI. At GSI the Lorentz factor is of the order of $\gamma \sim 1-2$. The equivalent photon spectrum contains energies up to several tens of $\mathrm{MeV}$ one can, with very large (of the order of one third) probability, excite the giant dipole resonance at an excitation energy of $80 A^{-1 / 3} \mathrm{MeV}$. Indeed, the excitation is so strong that also a two-photon transition can

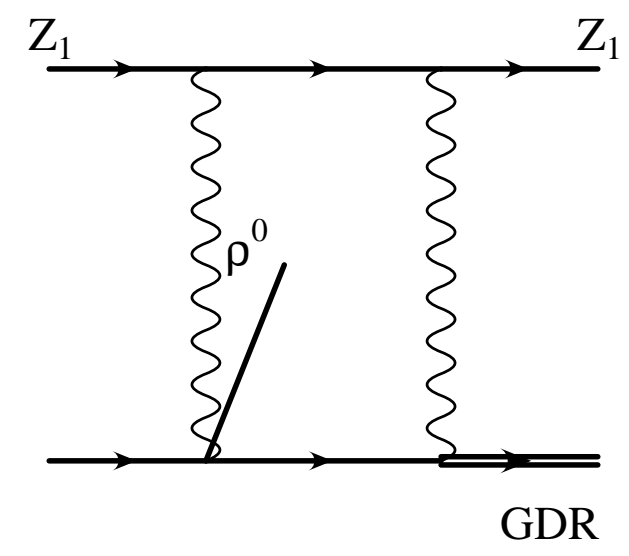

Fig. 3. Another second order graph. The final state is the same as in Fig.2.

occur to the higher harmonic mode, the double phonon giant dipole resonance [9, 10,11].

This GDR excitation is also present at the highest energies, at RHIC (Au-Au) and $\mathrm{LHC}(\mathrm{Pb}-\mathrm{Pb})$, where it causes a beam loss. It also serves a useful purpose: it is a trigger for UPC: in an ultraperipheral collision, the giant dipole resonance is excited, which decays mainly by neutron emission. These neutrons can be detected in the zero degree calorimeter. At RHIC $\rho^{0}$-mesons were produced in ultraperipheral collisions and detected with the STAR detector [12. At $\mathrm{LHC}(\mathrm{Pb}-\mathrm{Pb})$ the equivalent photon energy is much higher than at RHIC and one expects to produce and detect heavy vector mesons, possibly up to the $\Upsilon$ [13. Vector meson photoproduction is a useful tool for QCD studies [1, 14, 15].

In AA collisions the photon spectrum is enhanced by a factor of $Z^{2}$, on the other hand, the luminosity in pp collisions is appreciably higher as compared to AA. This compensates to a large extent, and also, due to the small size of the proton, the equivalent photon spectrum extends to even higher energies. At LHC(pp) electroweak and beyond the standard model physics studies are envisaged, see [16].

$\gamma-\gamma$ physics has been extensively studied at electronpositron colliders. $\gamma-\gamma$ collisions also occur in hadronhadron collisions. For heavy ions there is a $Z^{4}$-enhancement, on the other hand, the beam luminosity is typically rather low. The physics opportunities are described in [1], unfortunately the cross section for the production of a Higgs boson in photon-photon fusion is too low to be observable. In Fig. 4 a photon-photon collision is shown schematically. Electron-positron pairs are copiously produced, of the order of $100 \mathrm{~kb}$ at $\mathrm{LHC}(\mathrm{Pb}-\mathrm{Pb})$. The formation of positronium and muonium is discussed in Ch.7.7 of [17]. Below the threshold for pair production there is only elastic scattering, with a very small cross section with an $\omega^{6}$ rise. Despite the high photon fluxes, it seems hopeless to oberve this channel in heavy ion collisions. 


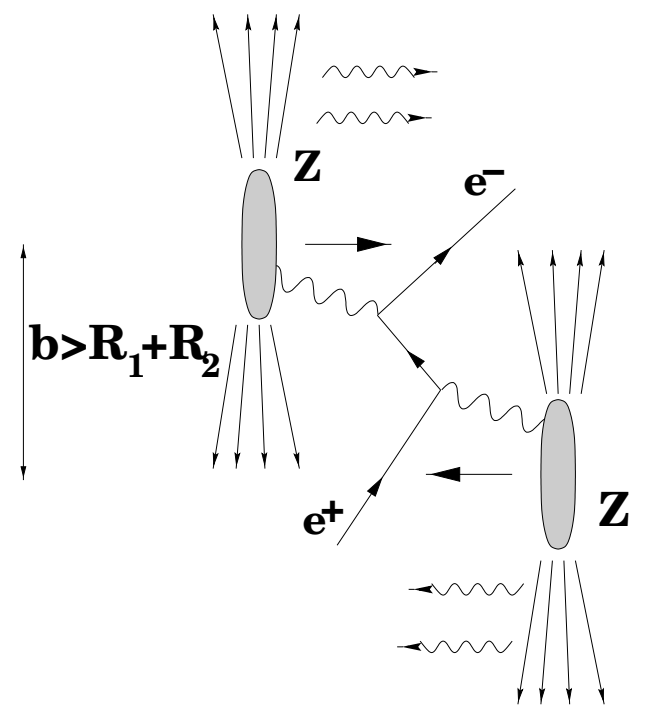

Fig. 4. A schematic picture of a photon-photon collision in an ultraperipheral heavy ion collision. Electron-positron pairs are produced with cross sections of the order of $100 \mathrm{~kb}$

\section{Higher order effects}

In lowest order Born approximation electron-positron pair production is given by the Racah formula

$$
\sigma_{\text {Racah }}=\frac{28 \alpha^{4} Z_{1}^{2} Z_{2}^{2}}{27 \pi m^{2}}\left(L^{3}-2.2 L^{2}+3.84 L-1.636\right) .
$$

where $L \equiv \ln \gamma^{2}$. This pair production process is closely related to the Bethe-Heitler process $\gamma+Z \rightarrow e^{+}+e^{-}+Z$, the real photon is now an equivalent photon, originating from the other nucleus. Coulomb corrections to the BetheHeitler formula were given by Bethe and Maximon. Quite analogously, there are Coulomb corrections in the heavy ion case, see 18, and the review 2] where further references can be found. Other higher order corrections are bound-free pair production and multiple pair production, which are briefly described in the following sections.

\subsection{Bound-free pair production}

Bound-free pair production is the process

$$
Z+Z \rightarrow\left(Z+e^{-}\right)_{\mathrm{K}-, \mathrm{L}-, \ldots \text { shell }}+e^{+}+Z
$$

where the electron is produced in a bound atomic orbit (K-,L-,..shell). The cross section scales approximately as

$$
\sigma \sim \frac{Z^{7} \ln \gamma \delta_{l 0}}{n^{3}}
$$

where $\mathrm{n}$ and $\mathrm{l}$ denote the principal and angular momentum quantum numbers of the atomic bound state. These ions with their changed charge-to mass ratio will get lost from the beam and they will heat up the beam pipe in a hot spot. It was identified as a serious limit for the luminosity in $\mathrm{Pb}-\mathrm{Pb}$ collisions at $\mathrm{LHC}$, due to the possible

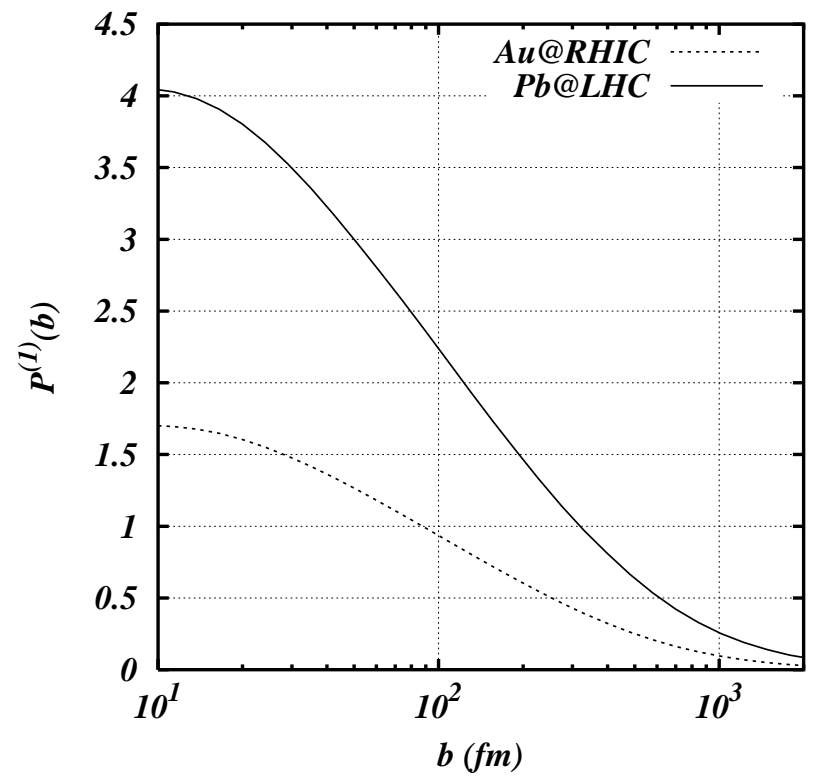

Fig. 5. Pair production probability calculated in lowest order Born approximation. Unitarity is violated manifestly. See text for restoration.

quenching of the superconducting magnets. The boundfree pair production cross section was recently measured at RHIC [19], see also 'The vacuum strikes back', Ref. [20]. Agreement with theory 21] is good. The bound-free pair production mechanism was also used in the production of fast antihydrogen in $\bar{p}-X e$-collisions at LEAR in 1996 by W. Oelert et al. 22 .

\subsection{Multiple pair production, a strong field effect}

The results of a lowest order calculation 23 of electronpositron pair production probability $P^{(1)}$ are shown in Fig. 5. For small impact parameters we have $P^{(1)}>1$ and unitarity is violated. In order to restore unitarity, one has to consider the production of multiple pairs in a single collision 24

$$
Z+Z \rightarrow n\left(e^{+}+e^{-}\right)+Z+Z
$$

One may treat the electron-positron pairs as quasibosons, a coherent state 25] of electron positron pairs is produced and one obtains a Poisson distribution for the production of $\mathrm{N}$ pairs $\mathrm{P}(\mathrm{N}, \mathrm{b}): P(N, b)=\frac{P^{(1)}(b)^{N}}{N !} \exp -P^{(1)}(b)$. This calculation bears some resemblance to the treatment of the infrared catastrophe in QED. Multiple pair production is a strong field effect. Although the cross section for multiple pair production is quite high, of the order of kilobarns, it will be difficult to observe these processes at the LHC due to the very low $p_{\perp}$ of most of the produced electrons and positrons.

\section{Conclusion}

The electromagnetic fields occurring in relativistic heavy ion collisions are extremely strong, they are well beyond 
the Schwinger critical field strength. However, these fields act only for an extremely short time. Perturbation theory (Feynman graphs) is an appropriate tool to describe these processes. Strong field effects manifest themselves in multi-photon excitation processes, like multiple electron positron pair production. The main interest is in the very high energy photons which occur due to the extremely short interaction time. This is in contrast to the physics of ultrahigh fields in laser physics.

\section{Acknowledgements}

I would like to thank Kai Hencken and Dirk Trautmann for their collaboration over many years. I am grateful to Dietrich Habs for interesting discussions and for inviting me to this stimulating workshop and school. I acknowledge the support by the European Commission under contract ELI pp 212105 in the framework of the program FP7 Infrastructures-2007-1.

\section{References}

1. A.J. Baltz et al. Physics Reports 458(2008)1

2. G.Baur, K.Hencken, and D.Trautmann, Physics Reports 453(2007)1

3. L.D. Landau, E.M. Lifshitz, Quantenelektrodynamik, Lehrbuch der Theoretischen Physik, No. IV, Akademie Verlag, Berlin, 1986

4. A.C. Melissinos, Spontaneous breakdown of the vacuum by intense lasers, in Electromagnetic probes of fundamental physics, Erice, Italy 16-21 October 2001, edited by W.Marciano and S.White, World Scientific, 2003

5. J.S. Schwinger, Phys. Rev. 76 (1949) 790

6. E.Brezin, C.Itzykson, Phys. Rev. D2 (1979) 1191

7. G.Baur, K.Hencken, A. Aste, D.Trautmann, and S.R. Klein, Nucl. Phys. A 729(2003) 787

8. K.Hencken, G.Baur, D.Trautmann, Phys. Rev. Lett. 96 (2006) 012303

9. J.Ritman et al. Phys. Rev. Lett. 70(1993)533

10. R.Schmidt et al. Phys. Rev. Lett. 70(1993)1767

11. K.Boretzki et al. Phys. Lett. B384(1996)30

12. B.I. Abelev et al. Phys. Rev. C77 (2008) 034910

13. J.Nystrand for the ALICE Collaboration, Nuclear Physics B (Proc. Suppl.) 179-180 (2008) 156, Proceedings of PHOTON-LHC-2008, CERN, Geneva, Switzerland, 22-28 April 2008

14. Mini-proceedings of the ECT* workshop on 'Photoproduction at collider energies: from RHIC and HERA to the LHC, arXiv: hep-ph/0702212

15. Workshop on 'High energy photon collisions at the LHC', 22-25 April 2008, CERN, Geneva, for slides see

http://indico.phys.ucl.ac.be/

conferenceDisplay $\cdot$ py? conf $I d=228$

Nucl. Phys. B (Proceedings Supplements) 179-180 (2008) PHOTON-LHC-2008

16. K. Piotrzkowski, Phys. Rev. D63(2001) 071502, K. Piotrzkowski, arXiv:0808.1624

17. G.Baur, K.Hencken, D.Trautmann, S. Sadovsky, Y. Kharlov Physics Reports 364 (2002) 359
18. D.Y. Ivanov, A. Schiller, V.G. Serbo, Phys. Lett. B454 (1999) 155

19. R.Bruce et al. Phys. Rev. Lett. 99 (2007) 144801

20. Physics News Update Number 841 http://www.aip.org/pnu/2007/split/841-1.html

21. H. Meier et al. Phys. Rev. A 63 (2001) 032713

22. G.Baur et al. Phys. Lett. B368 (1996) 251

23. K.Hencken, D.Trautmann, G.Baur Phys. Rev. A51 (1995) 1874

24. G.Baur, Phys. Rev. A42 (1990) 5736

25. R.Glauber, Phys. Rev. 131 (1963) 2766 